\title{
Pluripotent stem cells secrete Activin A to improve their epiblast competency after injection into recipient embryos
}

\author{
Jinzhu Xiang ${ }^{1}$, Suying $\mathrm{Cao}^{2}$, Liang Zhong ${ }^{1}$, Hanning Wang ${ }^{1,5}$, Yangli Pei ${ }^{1,6}$, Qingqing Wei ${ }^{1}$, Bingqiang Wen ${ }^{1}$, \\ Haiyuan Mu ${ }^{1}$, Shaopeng Zhang ${ }^{1}$, Liang Yue ${ }^{1}$, Genhua Yue ${ }^{3}$, Bing Lim ${ }^{4}$, Jianyong Han $^{1 凶}$ \\ ${ }^{1}$ State Key Laboratory for Agrobiotechnology, College of Biological Sciences, China Agricultural University, Beijing 100193, \\ China \\ ${ }^{2}$ Animal Science and Technology College, Beijing University of Agriculture, Beijing 102206, China \\ 3 Temasek Life Sciences Laboratory, National University of Singapore, Singapore 117604, Singapore \\ ${ }^{4}$ Stem Cell and Developmental Biology, Genome Institute of Singapore, Singapore 138672, Singapore \\ ${ }^{5}$ Beijing Advanced Innovation Center for Food Nutrition and Human Health, China Agricultural University, Beijing 100193, \\ China \\ ${ }^{6}$ State Key Laboratory of Animal Nutrition, Institute of Animal Sciences, Chinese Academy of Agricultural Sciences, Beijing \\ 100193, China \\ $\triangle$ Correspondence: hanjy@cau.edu.cn (J. Han)
}

Received June 26, 2017 Accepted August 22, 2017

\begin{abstract}
It is not fully clear why there is a higher contribution of pluripotent stem cells (PSCs) to the chimera produced by injection of PSCs into 4-cell or 8-cell stage embryos compared with blastocyst injection. Here, we show that not only embryonic stem cells (ESCs) but also induced pluripotent stem cells (iPSCs) can generate F0 nearly $100 \%$ donor cell-derived mice by 4-cell stage embryo injection, and the approach has a "dose effect". Through an analysis of the PSC-secreted proteins, Activin A was found to impede epiblast (EPI) lineage development while promoting trophectoderm (TE) differentiation, resulting in replacement of the EPI lineage of host embryos with PSCs. Interestingly, the injection of ESCs into blastocysts cultured with Activin A (cultured from 4-cell stage to early blastocyst at E3.5) could increase the contribution of ESCs to the chimera. The results indicated that PSCs secrete protein Activin A to improve
\end{abstract}

Co-first author: Jinzhu Xiang, Suying Cao, Liang Zhong, and Hanning Wang.

Electronic supplementary material The online version of this article (doi:10.1007/s13238-017-0470-y) contains supplementary material, which is available to authorized users. their EPI competency after injection into recipient embryos through influencing the development of mouse early embryos. This result is useful for optimizing the chimera production system and for a deep understanding of PSCs effects on early embryo development.

KEYWORDS pluripotent stem cells, 4-cell embryo injection, secreted proteins, Activin A, chimeric mice

\section{INTRODUCTION}

A conventional and useful approach for understanding gene function has involved producing genetically modified mice from embryonic stem cells (ESCs) that contain genetic changes since the successful derivation of mouse ESCs from blastocysts (D; Martin, 1981; Thomas and Capecchi, 1987). ESC-based transgenic mice are usually produced by the introduction of ESCs into diploid host embryos, generally blastocysts (Ramirezsolis et al., 1993; Stewart, 1993), resulting in chimeric mice that are only partially generated from ESCs. It takes a lot of time to produce homozygous mutant mice that are suitable for phenotyping. Previous reports have shown that the injection of ESCs into 4- or 8-cell stage embryos produces F0 nearly 100\% ESC-derived mice (ES-mice), with full germline transmission that permits immediate phenotypic analysis (Huang et al., 2008; 
Poueymirou et al., 2007). These methods significantly accelerated the process of gene function research.

Normal diploid embryos contribute to placental development and participate in fetal development (Rossant and Tam, 2009; Zernicka-Goetz et al., 2009). Injections of ESCs into diploid embryos could theoretically generate chimeric mice that are derived from both ESCs and host embryos. However, this outcome is not applicable to the injection of ESCs into the 4- or 8-cell embryos that can produce the ES-mice. The host embryo does not contribute to the fetal development in this method, which is similar to the tetraploid embryo that only contributes to extraembryonic tissues, such as the placenta (Eakin and Behringer, 2003). These results demonstrate that exogenous ESCs injected into 4- or 8-cell stage embryos have an impact on the host embryo developmental fate. Mouse ESCs injected into 8-cell stage embryos modified the pattern of cell lineage specification (Humiecka et al., 2016), which supports this view. However, it has not yet been completely elucidated how donor ESCs regulate the embryo developmental fate. Recent reports have indicated that ESCs or differentiating ESC-secreted proteins may affect the growth and development of exogenous cells, such as cell migration and myogenesis (Ngangan et al., 2014; Yousef et al., 2014). Therefore, we hypothesized that ESC-secreted proteins may affect the embryo developmental fate following the injection of ESCs into embryos.

Studies have shown that induced pluripotent stem cells (iPSCs) resemble ESCs in pluripotency, morphology, and differentiation abilities (Takahashi et al., 2007; Takahashi and Yamanaka, 2006). Notably, iPSCs could produce not only chimeric mice by blastocyst injection but also full-term offspring by tetraploid complementation (Kang et al., 2011; Maherali et al., 2007; Wernig et al., 2007; Zhao et al., 2009). Nevertheless, it remains unclear whether F0 nearly $100 \%$ iPSC-derived mice (iPS-mice) can be efficiently produced from 4- or 8-cell embryo injection.

In this work, we revealed that both ESCs and iPSCs could generate F0 nearly $100 \%$ donor cell-derived mice by the injection of cells into 4-cell stage embryos, and the 4-cell stage embryo injection assay had a "dose effect". In comparison to the injection of $20 \mathrm{ESCs}$, more coat chimeras were produced by the injection of fewer ESCs (i.e., 10 ESCs) into embryos. In addition, mouse ESC and iPSC-secreted protein Activin A was found to stunt epiblast (EPI) lineage development and to stimulate the development of the trophectoderm (TE) lineage in host embryos. We also showed that using embryos cultured with Activin A as the host increases the contribution of ESCs to the chimeras produced by conventional blastocyst injection. This process may explain the phenomenon that the injection of ESCs or iPSCs into 4-cell stage embryos yields F0 generation mice with a greater ESC or iPSC contribution than does blastocyst injection. The results indicated that PSCs secrete protein Activin A to improve their EPI competency after injection into recipient embryos via impacting on the development of mouse early embryos.

\section{RESULTS}

An increase in the number of cells injected into 4-cell stage embryos efficiently produces F0 generation ESmice or iPS-mice

In previous studies, we generated mouse iPS cell lines by different combinations of factors (Oct4, Sox2, Klf4 and Tbx3, termed OSKT; Sox2, KIf4 and Nr5a2, termed SKR; Sox2, $\mathrm{KIf} 4, \mathrm{Nr} 5 \mathrm{a} 2$ and c-Myc, termed SKRC) that were morphologically similar to mouse ESCs, were Oct4-GFP positive (Fig. 1A), could produce chimeras, and could even produce iPS-mice via tetraploid complementation assay (Han et al., 2010; Heng et al., 2010). We hypothesized that these cells could produce iPS-mice by injecting approximately 10 cells (9-11 cells) into 4-cell stage embryos based on previous reports (Huang et al., 2008; Poueymirou et al., 2007). While the injected embryos developed into blastocysts, these iPSCs could contribute to the inner cell mass (ICM) (Fig. 1B). The live mice produced by the 4-cell method were divided into three types: iPS-mice, chimeras, and host-derived mice (Fig. 1C). However, only a fewer iPS-mice were produced compared with wild-type R1 ESCs (Fig. 1D). To test whether the iPS-mice were chimeras, we randomly selected 2 and collected their tissues, such as heart, liver, spleen, lung and brain, to extract genomic DNA. We then used D12Mit60 primers to perform a microsatellite assay. Microsatellite analysis revealed that the iPS-mice generated by 4-cell embryo injection were all from iPSCs rather than host embryos (Fig. 1E and Table S1). To further detect the fate of iPSCs following injection into 4-cell stage embryos, we injected Oct4-GFP iPSCs into the embryos carrying actinGFP. The fluorescence location showed that Actin-GFP was negative in the fetus but positive in the placenta at embryonic day (E) 13.5. This result also demonstrates that iPSCs rather than host embryo EPI contributed to the fetus (Fig. 1F). An analysis of the gonads of high chimeric embryos further showed a high contribution of iPSCs with GFP expression to male and female gonads at E13.5, indicating that 4-cell stage embryo injection resulted in a high iPSC contribution to the gonad (Fig. 1G). We further identified that the adult iPS-mice exhibited 100\% germline transmission (Fig. 1H).

Interestingly, while increasing the injection cell numbers to approximately 20 , we found that these cells could efficiently produce ES-mice or iPS-mice, with coats and genotypes that were the same as the pluripotent stem cell background (Fig. 1C and 1D). This result suggests that the 4-cell stage embryo injection assay had a "dose effect" and that the injected pluripotent stem cells might force the host embryo blastomeres to change their fates, with the exogenous cells replacing the EPI to develop into a fetus. To confirm the conception, we used the G4-DsRed-MST ESCs (Vintersten et al., 2004) for the 4-cell embryonic injection assay (Fig. S1A and S1B). In addition to the evaluation of in vivo developmental potential, we performed immunofluorescent 


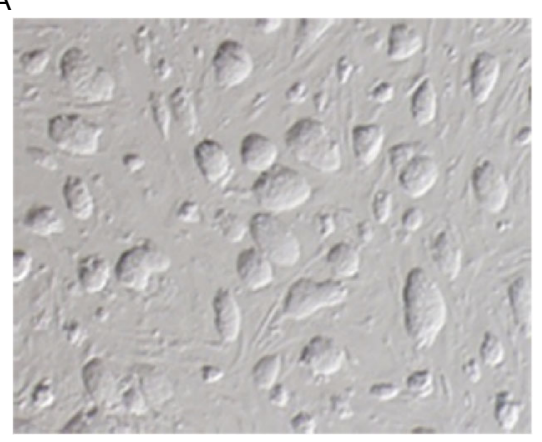

C

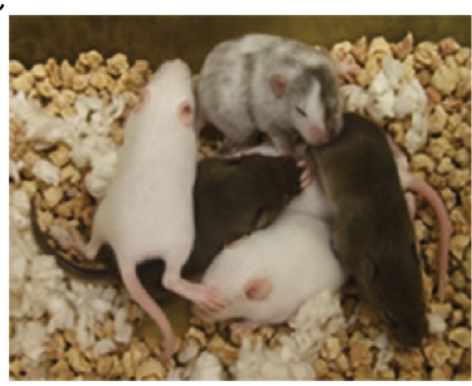

D
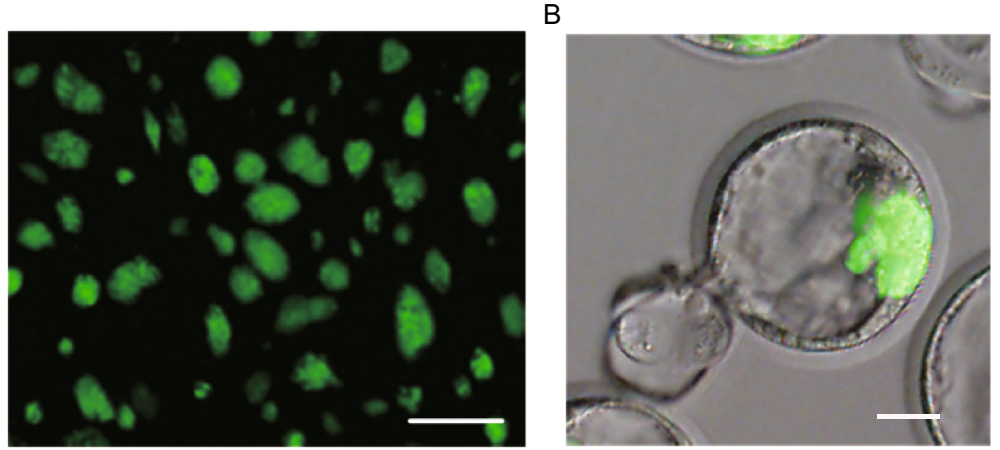

\begin{tabular}{|c|c|c|c|c|c|}
\hline \multirow{2}{*}{ PS cell line } & \multirow{2}{*}{$\begin{array}{c}\text { No. of } \\
\text { injected } \\
\text { cells }\end{array}$} & \multirow{2}{*}{$\begin{array}{c}\text { No. of the } \\
\text { reconstructed embryos/ } \\
\text { foster mother }\end{array}$} & $\begin{array}{c}|c| \\
\text { Offspring } \\
\text { Fo fully donor } \\
\text { cell- mice }\end{array}$ & Chimera & Wild type \\
\hline \multirow{2}{*}{ SKR B11 } & 10 & $40 / 3$ & 1 & 5 & 11 \\
\cline { 2 - 6 } & 20 & $75 / 3$ & 13 & 4 & 6 \\
\hline \multirow{2}{*}{ SKRC 1 } & 10 & $43 / 3$ & 0 & 4 & 7 \\
\cline { 2 - 6 } & 20 & $47 / 3$ & 8 & 6 & 4 \\
\hline \multirow{2}{*}{ OSKT 4-1 } & 10 & $45 / 4$ & 2 & 14 & 10 \\
\cline { 2 - 6 } & 20 & $40 / 3$ & 6 & 7 & 3 \\
\hline \multirow{2}{*}{ R1 ES } & 10 & $30 / 2$ & 4 & 3 & 3 \\
\cline { 2 - 6 } & 20 & $31 / 2$ & 6 & 1 & 2 \\
\hline
\end{tabular}

E

iPS

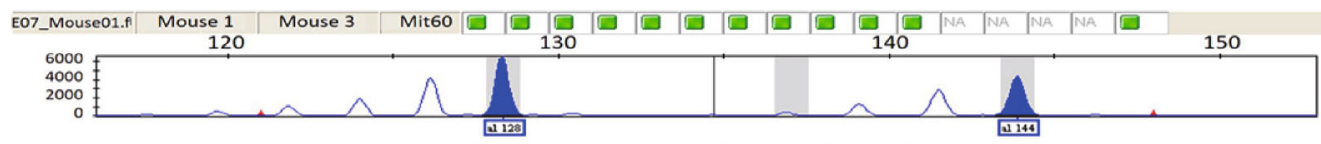

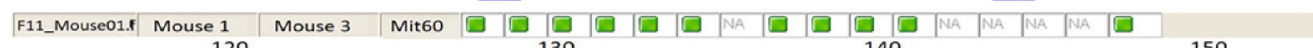

Embryo donor

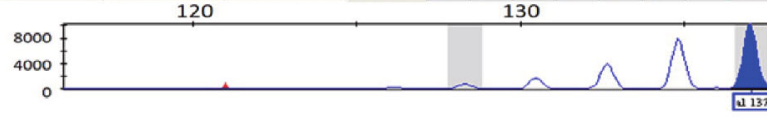

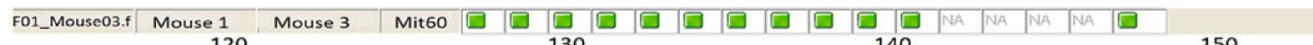

iPS-mice

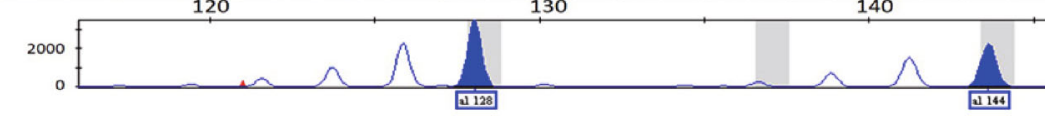

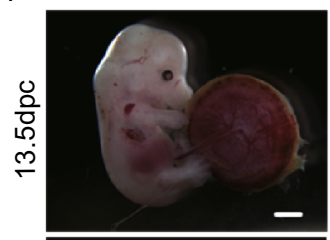

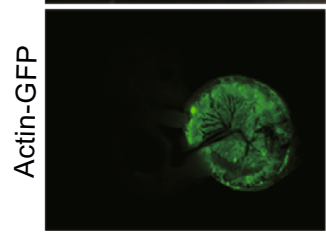

G

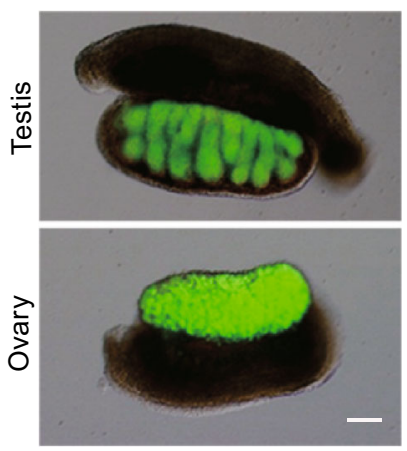

$\mathrm{H}$

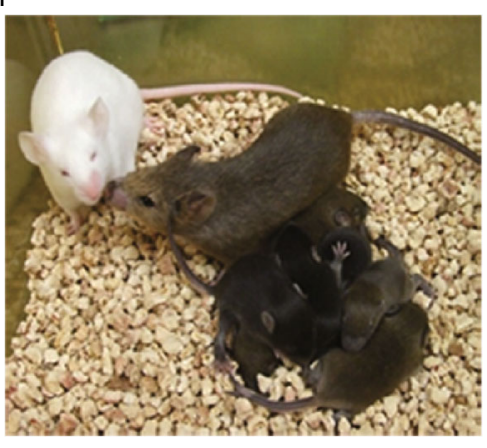

Figure 1. Generation of F0 iPS-mice by 4-cell stage embryo injection. (A) Mouse iPSC colonies (left panel, bright field; right panel, Oct4-GFP). Scale bar, $100 \mu \mathrm{m}$. (B) Blastocyst from 4-cell embryo injection. Scale bar, $20 \mu \mathrm{m}$. (C) F0 generation mice produced by 4 cell-stage embryo injection. (D) Summary of the generation of F0 fully iPSC or ESC-derived mice by 4-cell embryo injection of 20 or 10 donor cells. See also Fig. S1. (E) Representative microsatellite analysis of tails from iPSC-derived mice using D12Mit60 primers. See also Table S1. (F) Chimeric embryo at E13.5 produced by 4-cell stage embryo injection. Scale bar, $1 \mathrm{~mm}$. (G) Gonads of E13.5 embryos from 4-cell embryo injection. Scale bar, $200 \mu \mathrm{m}$. (H) Representative F0 iPSC-derived mouse (agouti color) and offspring. 
staining for the aggregation embryos at E4.5 to check Nanog localization in the ICM (Fig. S1A). In mouse embryo development, Nanog specifically expresses in the EPI, which gives rise to the future fetus, so Nanog staining can display the EPI cells (Rossant and Tam, 2009; Zernicka-Goetz et al., 2009). Immunofluorescent staining showed that EPI cells (defined on the basis of Nanog expression) were completely developed from ESCs in some blastocysts (Fig. S1C and S1D). As the numbers of injected ESCs increased, the percentage of blastocysts, whose EPI cells were only from ESCs, also increased. Of the blastocysts, 75\% (ESCsderived $\mathrm{EPI}$ ) were generated by the injection of 20 cells, and $31.25 \%$ of the blastocysts (ESCs -derived EPI) were derived by the injection of 10 cells (Fig. S1D). This result is consistent with the fact that F0 nearly $100 \%$ ESC and iPSC-derived mice can be produced by 4-cell stage embryo injection. This also suggests that donor ESCs impede the EPI lineage development of host embryos.

\section{ESC and IPSC secretions hinder EPI lineage development}

Cells can interact with each other through secreted factors. Many reports have shown that ESCs secrete cytokines and proteins that can affect the fate of other cells around them (Ngangan et al., 2014; Yousef et al., 2014). Therefore, the secretions of ESCs and iPSCs, which were injected into the 4-cell stage embryos, might hinder the EPI lineage specification during further development. To confirm this hypothesis, we chose the ESC and iPSC lines, which can produce ES-mice or iPS-mice, to collect the condition medium and to explore their effects on the EPI development of preimplantation embryos after culture in vitro (Fig. 2A). Zona-free embryos at the 4-cell stage were cultured in the mixed medium containing the condition medium and KSOM (1:1) (Fig. 2B). When 4-cell embryos in the mixed medium developed into E4.5 blastocysts, cell numbers of the EPI lineage (Nanog-positive cells) were detected by immunofluorescent staining. ESCs and iPSCs were maintained on feeder cells, so condition medium collected from feeder cells only was used as the control group. The results showed that a decline in the Nanog expression level was apparent (Fig. 2C), and that the EPI cell numbers were significantly reduced (Fig. 2D) in the blastocysts treated by the mixed medium, including KSOM and the condition medium from the R1 ESCs or iPSCs. These results indicate that ESC and iPSC secretions indeed suppress EPI lineage development.

\section{ESC and iPSC-secreted protein Activin A impedes the development of EPI lineage}

To test the components of the condition medium, we performed mass spectrometry and then obtained a list of candidate proteins (Fig. 2E). After screening, we found that Nanog expression significantly declined and EPI cell numbers decreased in Activin A-treated embryos when its concentration was $500 \mathrm{ng} / \mathrm{mL}$ (Fig. $3 \mathrm{~A}$ and $3 \mathrm{~B}$ ), indicating that Activin A works as a member of secreted proteins during EPI development similar to that in the condition medium. As its concentration was reduced to $100 \mathrm{ng} / \mathrm{mL}$, the effect abated. By contrast, the effect was strengthened but not obvious as the concentration increased up to $3,000 \mathrm{ng} / \mathrm{mL}$ (data not shown). Hence, Activin $A$ at a concentration of $500 \mathrm{ng} / \mathrm{mL}$ was used for subsequent experiments.

Activin $A$ is a member of the transforming growth factor $\beta$ (TGF $\beta$ ) superfamily. Activin A exerts biological effects by interacting with its receptors, including ACVRIIA, ACVRIIB, ALK4, and ALK7 (Pauklin and Vallier, 2015). We found that the expression of Activin A receptors were higher in 4-cell embryos than in blastocysts (Fig. S2) by analyzing the published data (Fan et al., 2015). SB431542 is a potent and specific inhibitor of transforming growth factor-superfamily type I Activin receptor-like kinase (ALK) receptors such as ALK4, ALK5, and ALK7 (Inman et al., 2002). To determine whether the inhibitor affects EPI development, 4-cell embryos were cultured in the medium containing $10 \mu \mathrm{mol} / \mathrm{L}$ SB431542 until they developed into late blastocysts. EPI cell numbers were detected by immunofluorescent staining. The Nanog-expressing cells were increased compared with the control group, showing that more EPI cells appeared in the SB431542 group (Fig. S3A and S3B). This result is in agreement with the previous report (Ghimire et al., 2015) and further proves the effect of Activin A on early embryo development.

Next, to further confirm the impact of Activin A on early embryo development, we analyzed the transcriptomes of embryos treated with Activin A (Fig. 3C) and compared them with the control group. The analysis results showed that the expression levels of EPI marker genes such as Pou5f1, Nanog, and Sox2 were decreased in embryos in the Activin A group (Fig. 3D), which was corroborated by the quantitative RT-PCR assay (Fig. 3E). These results indicate that Activin $A$ hinders the development of the EPI lineage.

ESCs were derived from the EPI lineage in blastocysts (Evans and Kaufman, 1981; Martin, 1981). Activin A reduced the numbers of EPI cells as shown above, so we wonder whether Activin A affects the success rate of ESC isolation. Zona-free 4-cell embryos were cultured in $500 \mathrm{ng} / \mathrm{mL}$ Activin A until E4.5 and then seeded on feeder cells containing mouse ESCs medium plus PD0325901 and CHIR99021 (2i) (Fig. 4A). Eight days later, we examined numbers and areas of Nanog-positive outgrowths (Fig. 4A). Interestingly, the ratio of Nanog-positive outgrowths in the Activin A treatment group was significantly lower (Fig. 4B), and the areas of Nanog-positive colonies were smaller than those of the control group (Fig. 4C and 4D). These results demonstrate that Activin A has a negative effect on EPI cell development and also affects the isolation and primary cloning of mouse ESCs. 
A

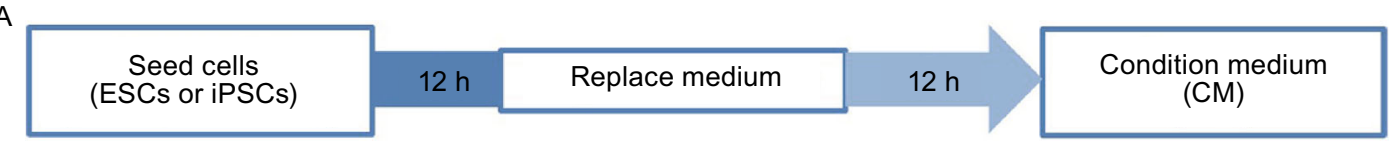

B

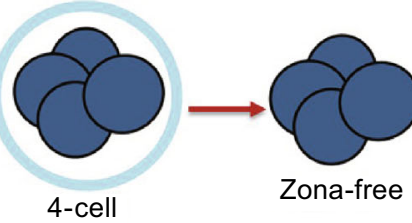

C
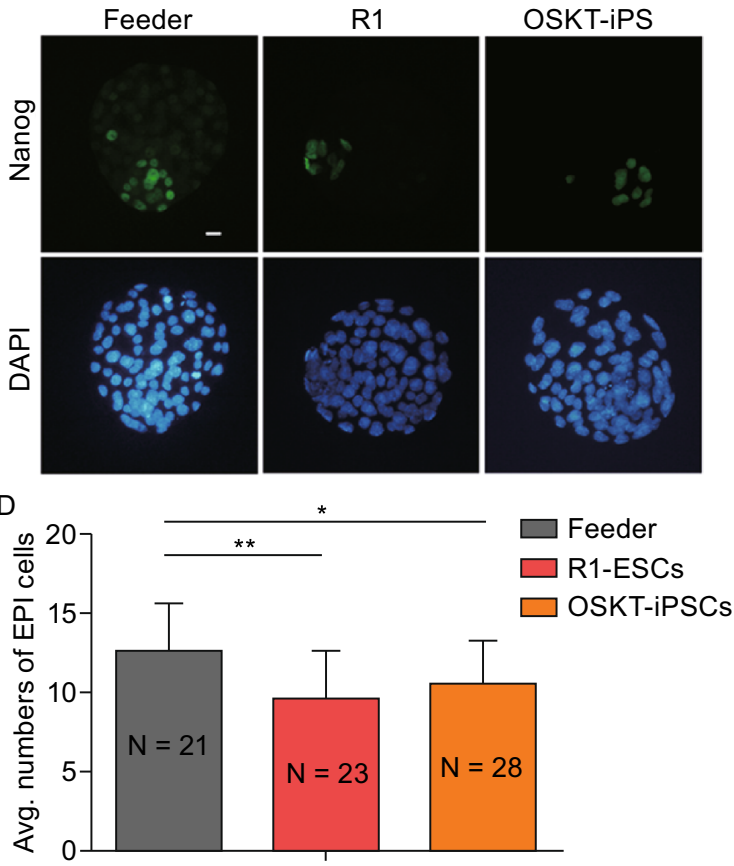

R1

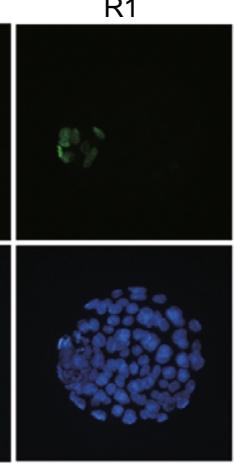

OSKT-iPS

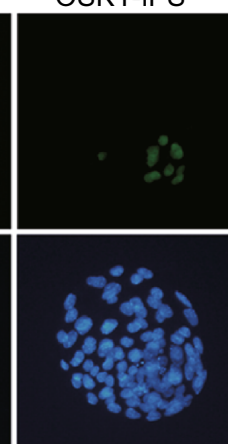

$\mathrm{CM}: \mathrm{KSOM}=1: 1$

$$
\text { Blastocyst }
$$

E

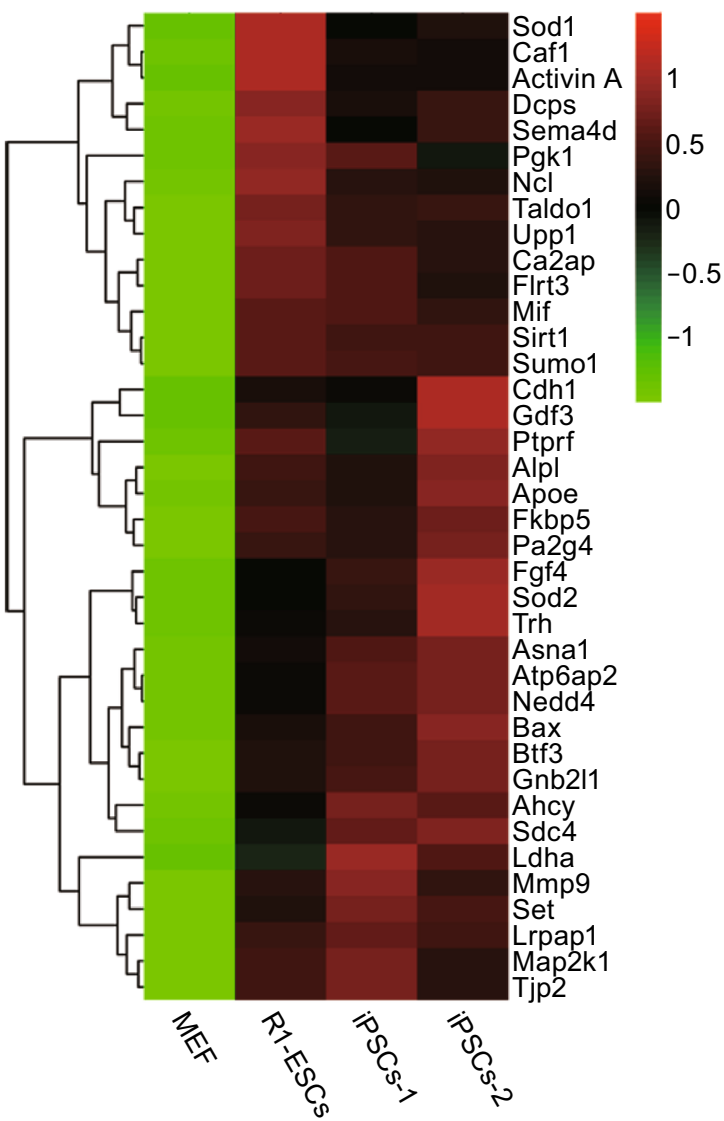

Figure 2. Secretions from ESCs and iPSCs affect EPI development. (A) Schematic of the method used to collect the condition medium. (B) Experimental design. Zona-free embryos at 4-cell stage were treated in the mixed medium containing KSOM and CM and then immunostained at E4.5 to test the effect of the condition medium on early embryo development fate. CM, condition medium. (C) Nanog immunostaining in E4.5 embryos treated with condition medium from feeder, R1 ESCs and iPSCs. Nuclei were stained with DAPI (Blue). Scale bars, $20 \mu \mathrm{m}$. (D) Average numbers of EPI cells (Nanog-positive cells) in condition medium-treated embryos at E4.5. Error bars indicate SD. ${ }^{*} P<0.05 ;{ }^{\star} P<0.01$ by ANOVA. $N$ is the number of embryos examined. (E) Heatmap of ESC and iPSCsecreted proteins at high expression levels. The heatmap was plotted with relative protein expression.

\section{ESC and iPSC-secreted protein Activin A promotes TE lineage development}

There are two other lineages, TE and primitive endoderm (PE), in addition to the EPI lineage in the late blastocyst (Cockburn and Rossant, 2010; Wang and Dey, 2006). We hypothesized that the secreted protein Activin A might promote embryo blastomeres toward TE or PE lineages, and we further analyzed the RNA-Seq data. A heatmap showed that the expression levels of TE marker genes such as Id2 and Cdx2 were elevated in the Activin A group (Fig. 5A). These data were consistent with the quantitative RT-PCR results (Fig. 5B). Immunofluorescence staining also revealed that the TE marker protein CDX2 was clearly elevated in embryos following treatment with Activin A (Fig. 5C and 5D). These findings indicate that Activin A promotes TE lineage 
A

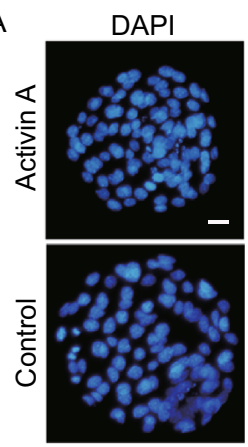

$\mathrm{D}$

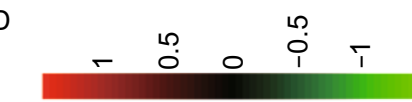

B

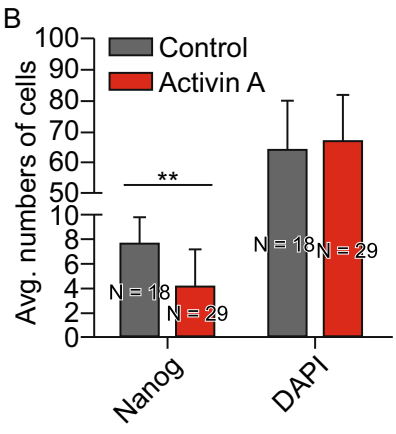

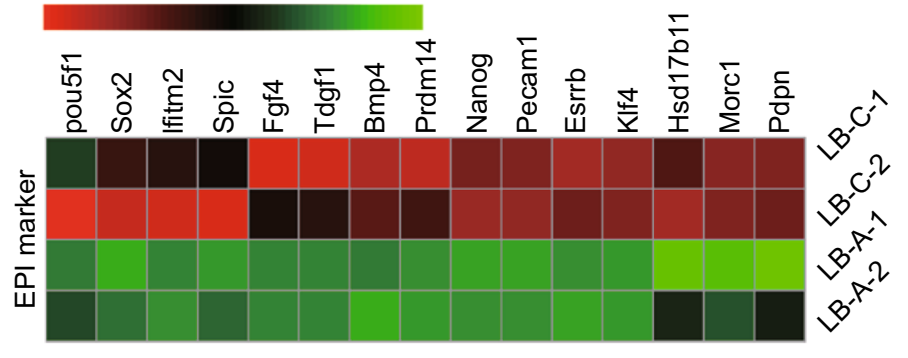

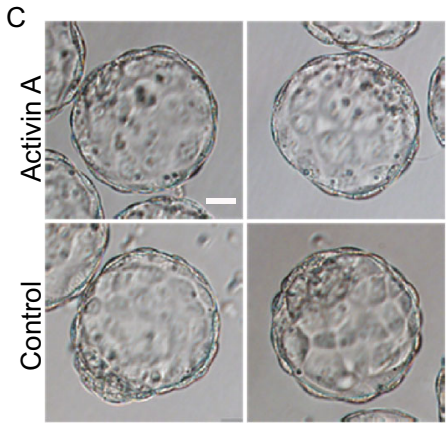

$\mathrm{E}$

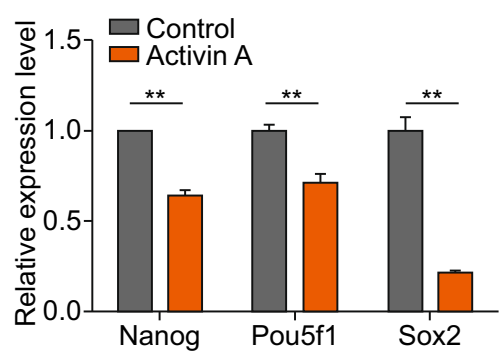

Figure 3. Activin A represses EPI lineage. (A) Nanog immunostaining in Activin A-treated and untreated embryos at E4.5. Nuclei were stained with DAPI (Blue). Scale bars, $20 \mu \mathrm{m}$. (B) Average numbers of EPI cells (Nanog-positive cells) in Activin A-treated and untreated embryos at E4.5. Error bars indicate SD. ${ }^{* *} P<0.01$ by ANOVA. $N$ is the number of embryos examined. (C) Late blastocysts in the Activin A-treated group and the control group. Scale bars, $20 \mu \mathrm{m}$. (D) Heatmap of selected genes associated with EPI lineage. Heatmap was plotted with Z-score normalized RPKM. (E) Quantitative RT-PCR analyzed the expression of genes selected by heatmap of EPI Markers. Error bars indicate SD $(n=3) .{ }^{* \star} P<0.01$ by Student's $t$-test.

development and impedes EPI lineage development. However, our analysis data showed that Activin A has no obvious effects on PE lineage development.

Increase in the contribution of ESCs to ICM and chimeras using Activin A-treated early blastocysts as recipients

We next performed the blastocyst injection assay to further examine the role of ESC-secreted proteins in chimeras producing. Activin A-treated blastocysts (cultured from 4-cell stage to early blastocyst at E3.5) were taken as host embryos, and G4-DsRed-MST ESCs were taken as donor cells (Fig. 6A and 6B). We detected the fates of ESCs that were injected into blastocysts when chimeric embryos developed to the late blastocyst stage. Fluorescent locations of ESCs and immunostaining of Nanog indicated that $38.7 \%$ of aggregated embryos showed a high contribution of ESCs to the EPI lineage in host embryos treated by Activin A compared with embryos in the control group (15\%) (Fig. 6C-E). Additionally, we detected the E10.5 chimeric embryos after embryo transplantation, which showed that Activin A treatment increased the average contribution of ESCs to the chimeric fetus (Fig. 6F and 6G) and increased the rate of higher ESC-contributed chimeras $(25 \%-75 \%$ and $>75 \%$ ESCs contribution) while decreasing the rate of lower ESC-contributed chimeras ( $<25 \%$ ESCs contribution) (Fig. $6 \mathrm{~F}$ and $6 \mathrm{H}$ ). The results were similar to those in the E4.5 chimeras mentioned above. Taken together, the analysis and comparison of the ESC contribution in chimeric embryos at different stages demonstrate that the ESC-secreted protein Activin A following treatment for host embryos can increase the contribution of ESCs to the chimeras produced by blastocyst injection by impeding EPI lineage development of the host embryos.

\section{DISCUSSION}

The injection of ESCs into 4-cell stage embryos resulted in higher ESC contributions to the chimera than the blastocyst-based approach (Huang et al., 2008; Poueymirou et al., 2007). One possible reason for this difference is that exogenous ESCs have some effects on early embryo development. As reported, ESCs decrease the cell numbers of the ICM of host embryo-origin during blastocyst formation (Humiecka et al., 2016). However, there are different views about how ESCs exert regulatory roles in mouse early embryo development. One theory is that ESCs influence blastomere division (Humiecka et al., 2016), while another is that ESCs have a competitive advantage compared with early blastomeres (Poueymirou et al., 2007). 
A
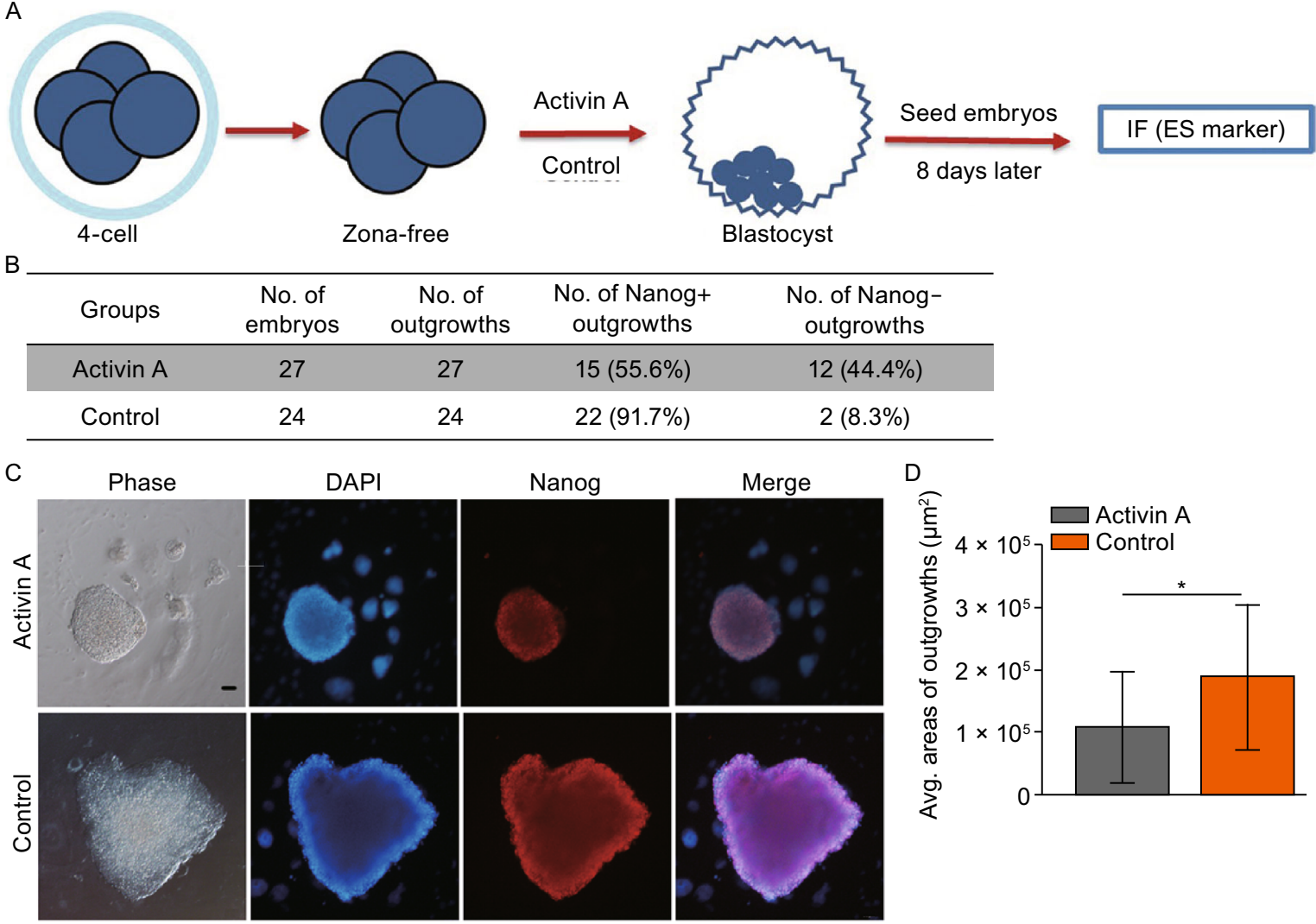

Figure 4. Activin A has a negative effect on the derivation of ESCs. (A) Experimental design for outgrowth analysis. Activin A treated-embryos were seeded on the feeder cells at $E 4.5$ and then immunostained 8 days later to test whether Acticin $A$ has effects on ES cell isolation. (B) Summary of outgrowths derived from Activin A treated and untreated late blastocysts. $P<0.05$ by Chi-square test. (C) Nanog immunostaining in outgrowths derived from Activin A treated and untreated blastocysts. Nuclei were stained with DAPI (Blue). Scale bars, $50 \mu \mathrm{m}$. (D) Average areas $\left(\mu \mathrm{m}^{2}\right)$ of Nanog positive outgrowths. Error bars indicate SD. ${ }^{*} P<0.05$ by ANOVA.

At the onset of our study, we hypothesized that ESCs or iPSCs influence the development of early embryos by secreting certain factors. We confirmed that FO ESC or iPSC-derived mice could be generated by the injection of approximately 10 cells into a 4-cell stage embryo. A previous study reported that the injection of more than 9 cells through the laser-assisted injection system has no added benefit because the excess cells tend to leak out through the opening in the zona pellucida (Poueymirou et al., 2007). Using the Piezo Micro Manipulator (PMM-150), we can easily inject 20 cells into one embryo, and we found that the injection of 20 iPSCs or ESCs instead of 10 cells into embryos increased the probability of obtaining F0 generation ES-mice or iPS-mice. We then explored the evidence supporting that ESC and iPSC-secreted proteins can improve their EPI competency after injection into recipient embryos. The key protein, Activin A, plays a positive role in generating chimeras with a higher degree of ESC contribution via impeding the EPI lineage development of host embryos. This can be explained by the results that numbers of EPI cells decreased during the formation of the blastocysts cultured with the condition medium from ESCs and iPSCs or cultured with Activin A (Figs. 2 and 3). These results indicated that the ESC or iPSC-secreted protein Activin A hinders EPI lineage development. Because of the small number of EPI cells, a high-ratio chimera could be generated after injection of the same number of pluripotent stem cells into recipient embryos, which increased the ratio of exogenous cells in the ICM. This conclusion was also supported by the transcriptome of the blastocysts treated with Activin A from the 4-cell stage (Fig. 3). Transcription factors such as Pou5f1 and Nanog, which are key regulators during EPI specification, were significantly reduced after treatment with Activin A. A previous report, which supports our conclusion, showed that Activin A can hinder EPI formation in early mouse embryos (Ghimire et al., 2015). These associations were further confirmed by our result that the cell number of EPI increased in the presence of the Activin receptor inhibitor SB431542 (Fig. S3). These data clearly demonstrate that ESC-secreted proteins facilitate the production of a higher ESC contribution 
to chimeras by impeding the development of EPI cells in host embryos.

We further revealed that Activin A increased the numbers of the TE lineage (Fig. 5), which may provide clues to explain the effects of Activin A or even TGF- $\beta$ signaling pathways on the preimplantation embryonic development. Nodal/Activin signaling has been shown to be required for trophoblast stem cell (TSC) renewal in culture conditions, while working with other factors (Guzman-Ayala et al., 2004; Ohinata and Tsukiyama, 2014). This result can explain why the TE or TSC marker gene expression level increased in the blastocysts treated with Activin A, while the PE-related genes remain comparable. Using Activin A-treated embryos as hosts enhances the ESC contribution to the chimeras produced by the traditional blastocyst-based approach. This result further confirmed the role of Activin $A$ in preimplantation embryos, as well as in the 4-cell embryo injection assay. This process will have great potential to efficiently produce gene-edited mice.

In summary, we conclude that the ESC and iPSC secrete protein Activin A to improve their EPI competency after injection into recipient embryos through impacting on the development of early embryo. Our study not only gives an
Figure 6. Activin A enhances ESC contribution to chimeras generated by blastocyst injection. (A) Experimental design. We treated 4-cell embryos without zona in KSOM including Activin $A$ and then performed the blastocyst injection to test whether Activin A can affect the contribution of ESCs to chimeras. (B) The ESC colonies (left panel, bright field; right panel, DsRed). Scale bar, $20 \mu \mathrm{m}$. (C) ESC-injected embryos at E4.5. Scale bar, $20 \mu \mathrm{m}$. (D) Nanog immunostaining in E4.5 chimeric embryos after blastocyst injection. Nuclei were stained with DAPI (Blue). Scale bars, $20 \mu \mathrm{m}$. (E) Summary of ESC (DsRed) contribution to E4.5 embryos. $P<0.05$ by Chisquare test. The high group indicates that DsRed ESCs almost overlaid Nanog-positive cells as shown in the top panel of Fig. 4D, and others are included in the low group.

(F) Chimeric embryos at E10.5. Scale bar, $500 \mathrm{~mm}$.

(G) Average ESC contribution to chimeric embryos at E10.5 using ImageJ software. ${ }^{*} P<0.05$ by ANOVA. (H) Summary of ESC (DsRed) contribution to E10.5 chimeric embryos. $P<0.05$ by Chi-square test.

effective explanation for the higher contribution of pluripotent stem cells to the EPI component and chimeras produced by the 4-cell approach compared with blastocyst injection, but
A

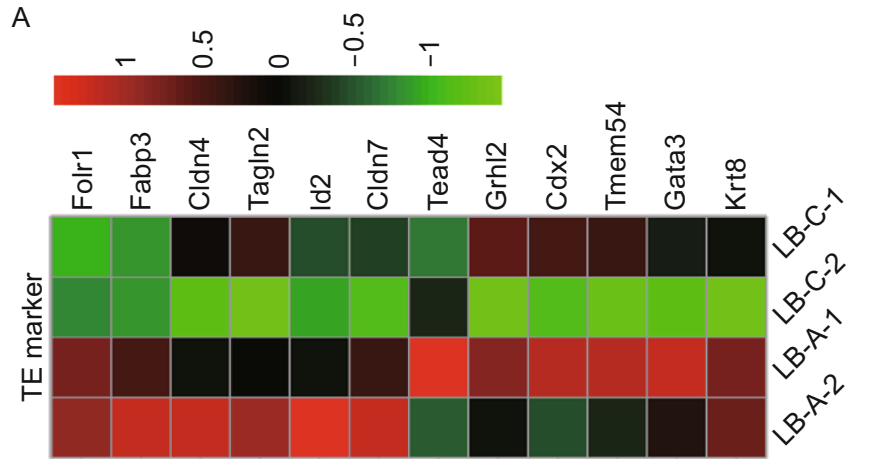

C
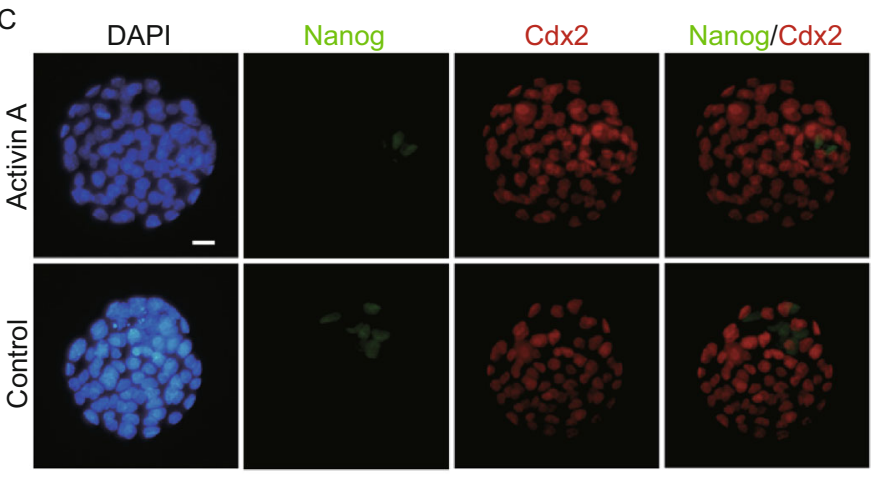
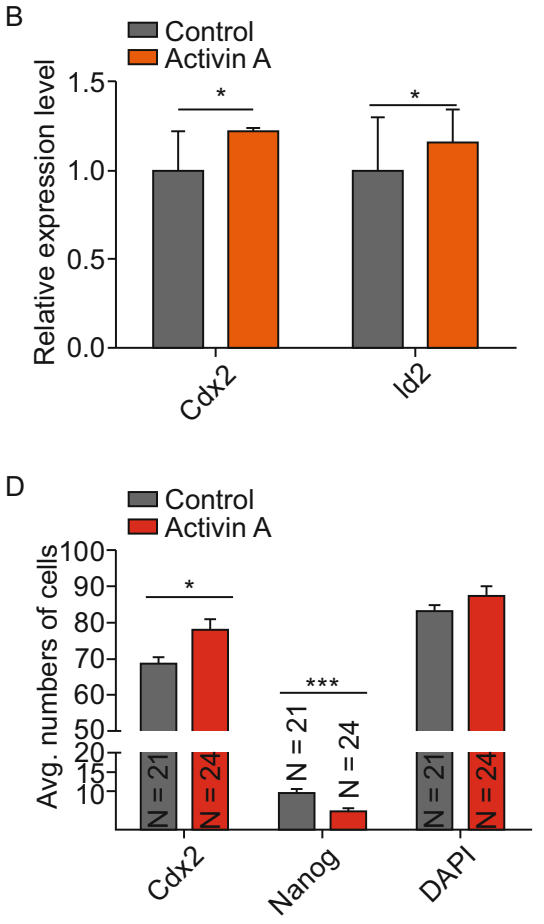

Figure 5. Activin A promotes TE lineage. (A) Heatmap of selected genes related to TE lineage. Heatmap was plotted with Z-score normalized RPKM. (B) Quantitative RT-PCR analyzed the expression of genes selected by heatmap of TE Markers. Error bars indicate SD $(n=3)$. ${ }^{*} P<0.05$ by Student's $t$-test. (C) Cdx2 immunostaining in Activin A-treated and untreated embryos at E4.5. Nuclei were stained with DAPI (Blue). Scale bars, $20 \mu \mathrm{m}$. (D) Average numbers of TE cells (Cdx2-positive cells) and EPI (Nanog-positive cells) in Activin A-treated and untreated embryos at E4.5. Error bars indicate SD. ${ }^{*} P<0.05,{ }^{* \star *} P<0.001$ by ANOVA. N is the number of embryos examined. 


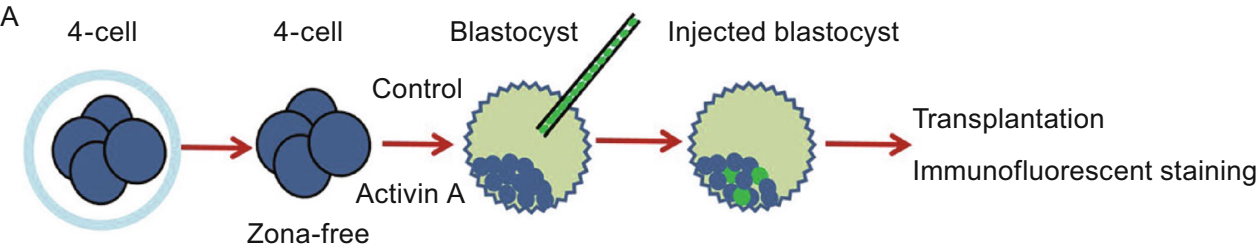

B
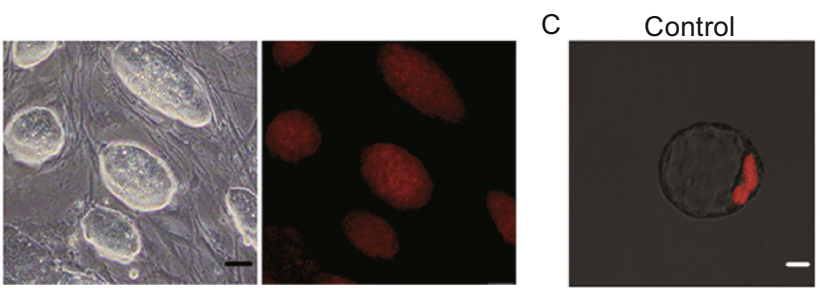

Activin A

D

DAPI
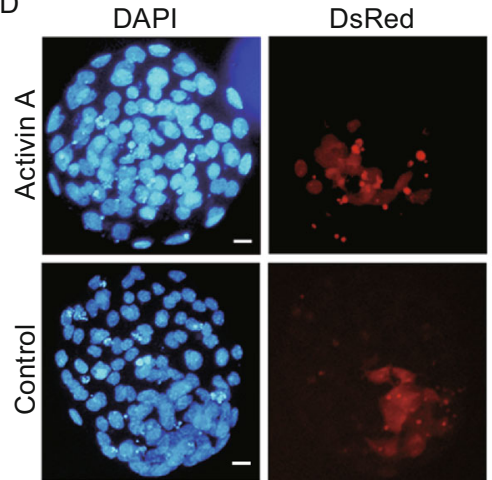

E

\begin{tabular}{ccccc}
\hline \multirow{2}{*}{ Groups } & $\begin{array}{c}\text { Injected } \\
\text { embryos }\end{array}$ & $\begin{array}{c}\text { DsRed+ } \\
\text { embryos }\end{array}$ & \multicolumn{2}{c}{ Contribution of DsRed cells to EPI } \\
\cline { 4 - 5 } & & 31 & High & Low \\
\hline Activin A & 31 & $12(38.7 \%)$ & $19(61.3 \%)$ \\
Control & 41 & 40 & $6(15 \%)$ & $34(85 \%)$ \\
\hline
\end{tabular}

F Phase
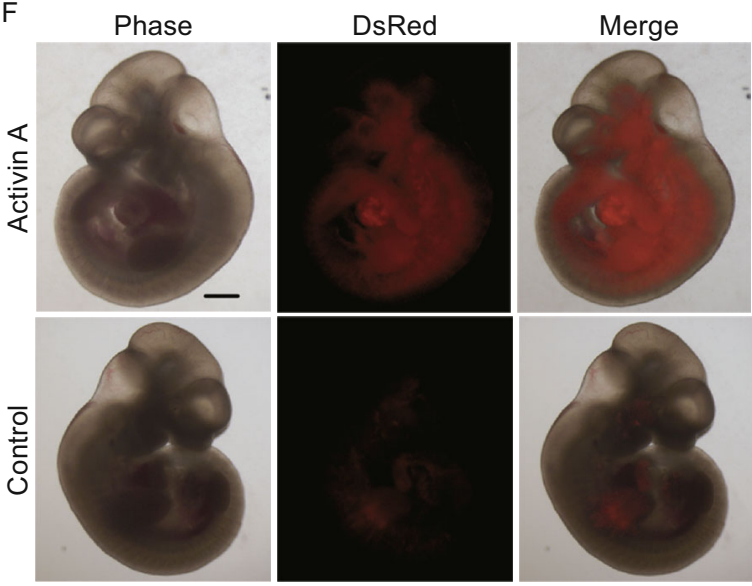

G
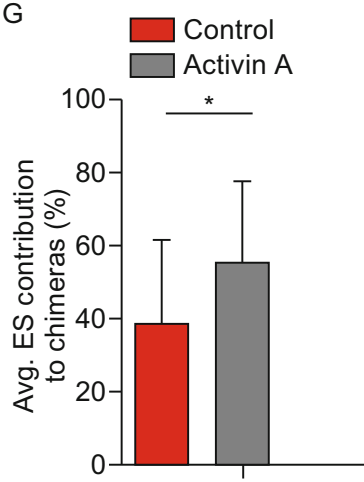

$\mathrm{H}$

\begin{tabular}{cccccc}
\multirow{2}{*}{ Groups } & \multirow{2}{*}{$\begin{array}{c}\text { Injected } \\
\text { embryos }\end{array}$} & $\begin{array}{c}\text { DsRed+ } \\
\text { embryos/Total }\end{array}$ & \multicolumn{2}{c}{ Contribution of DsRed cells to embryos } \\
\cline { 4 - 6 } & & $19 / 25$ & $<25 \%$ & $25 \%-75 \%$ & $>75 \%$ \\
\hline Activin A & 143 & $19.3 \%)$ & $14(73.7 \%)$ & $4(21 \%)$ \\
Control & 132 & $23 / 25$ & $10(43.5 \%)$ & $11(47.8 \%)$ & $2(8.7 \%)$ \\
\hline
\end{tabular}


also provides a new idea and theoretical basis for the optimization of chimera production systems in the future.

\section{MATERIALS AND METHODS}

\section{Animal experiments}

All animal studies proceeded according to the guidelines of the Institute Animal Care and Use Committee and were approved by the Animal Care and Use Committee of China Agricultural University. We used CD1 (ICR) mice as the embryo donors and recipients, which were purchased from Beijing Vital River Laboratory Animal Technology Co., Ltd. (Beijing, China). All mice were maintained in specific pathogen-free (SPF) conditions with a 12-h dark/12-h light cycle.

\section{Cell culture}

The ESCs and iPSCs were maintained on mitomycin C-treated mouse embryonic fibroblast (MEF) feeder cells in the ESC medium. The ESC medium contained DMEM (Invitrogen) with 15\% FBS, $2 \mathrm{mmol} / \mathrm{L}$ GlutaMAX, $1 \mathrm{mmol} / \mathrm{L}$ sodium pyruvate, $2 \mathrm{mmol} / \mathrm{L}$ nonessential amino acids, $0.1 \mathrm{mmol} / \mathrm{L}$ 2-mercaptophenol (all from Gibco), and 1000 units/mL LIF (Millipore).

\section{Condition medium collection and mass spectroscopy}

The initial cells (ESCs and iPSCs) were seeded at a density of $5.0 \times 10^{5}$ cells per well of a 6 -well plate on feeder cells. The original medium was replaced with serum-free fresh ESC medium $12 \mathrm{~h}$ later. The supernatant was collected and then centrifuged at 4,000 rpm for $60 \mathrm{~min}$ at $4^{\circ} \mathrm{C}$ after $12 \mathrm{~h}$. The supernatant was stored at $-80^{\circ} \mathrm{C}$ before further processing, such as mass spectrometry.

Liquid chromatography-tandem mass spectrometry (LC-MS/MS) analysis was carried out by Capitbalbio Technology using the $Q$ Exactive mass spectrometer (Thermo Scientific, CA). Mass spectrometry analysis was performed in a data-dependent manner, with full scans $(350-1,600 \mathrm{~m} / \mathrm{z})$ acquired using an Orbitrap mass analyzer at a mass resolution of 70,000 at $400 \mathrm{~m} / \mathrm{z}$ in $Q$ Exactive.

\section{Embryo collection and culture in vitro}

To obtain 2-cell stage embryos, female mice were superovulated by intraperitoneal injection of 5 international units (IU) of PMSG, followed by 5 IU HCG $46-48 \mathrm{~h}$ later, and mated with male mice. 2-cell stage embryos were obtained by flushing the oviduct with M2 at E1.5. Embryos were washed in M2 (Millipore) and then transferred into $10 \mu \mathrm{L} \mathrm{KSOM}$ (Millipore) drops covered with mineral oil (Sigma) on a tissue culture dish. The embryos were maintained at $37^{\circ} \mathrm{C}$ with $5 \% \mathrm{CO}_{2}$ in an incubator (Thermo Scientific).

For SB431542-treated embryos, 4-cell embryos were cultured in KSOM with $10 \mu \mathrm{mol} / \mathrm{L}$ SB431542, and embryos in the control group were cultured with an equivalent amount of DMSO in KSOM.

For condition medium-treated embryos, the zonas of 4-cell embryos were removed by acidic Tyrode's solution (Millipore). Zonafree 4-cell embryos were cultured in the mixed medium including $50 \% \mathrm{KSOM}$ and $50 \%$ condition medium from ESCs and iPSCs. Zona-free embryos at the 4-cell stage in the control group were cultured in the mixed medium, including $50 \% \mathrm{KSOM}$ and $50 \%$ condition medium from feeder cells. Embryos were fixed at E4.5 for EPI detection.

For Activin A-treated embryos, zona-free 4-cell embryos were cultured in $\mathrm{KSOM}$ with $500 \mathrm{ng} / \mathrm{mL}$ Activin A. Zona-free embryos at the 4-cell stage in the control group were cultured with an equivalent volume of $0.1 \%$ BSA in KSOM. Embryos were fixed at E4.5 for EPI detection.

For outgrowth assays, embryos were cultured in KSOM with Activin $A$ as above until E4.5 and then transferred on feeder cells in the ESC medium plus CHIR99021 and PD0325901 (Selleck). After treating for 8 days, outgrowths were fixed for immunofluorescence staining.

\section{Immunofluorescence staining}

Embryos were fixed with $4 \%$ paraformaldehyde in DPBS for $30 \mathrm{~min}$ at room temperature and then washed three times with $0.2 \% \mathrm{BSA}$ in DPBS. Embryos were then permeabilized at room temperature in $0.5 \%$ Triton X-100 in DPBS for $30 \mathrm{~min}$. After washing according to the above method, embryos were incubated with primary antibodies in $0.2 \% \mathrm{BSA}$ for $4 \mathrm{~h}$ at room temperature, washed with $0.2 \% \mathrm{BSA}$ and incubated with secondary antibodies in $0.2 \%$ BSA for $1 \mathrm{~h}$ at room temperature. Following washing with $0.2 \%$ BSA, the embryos were incubated for $3 \mathrm{~min}$ in the mounting medium with DAPI.

Immunostaining was performed with Nanog (Cell Signaling Technology, 1:500) and Cdx2 primary antibodies (Biogenex, 1:200). The secondary antibodies used in this research were as follows: Alexa594 goat anti-rabbit IgG, Alexa488 goat anti-rabbit IgG, and Alexa594 donkey anti-mouse IgG antibodies (Invitrogen, 1:500).

\section{ESC or iPSC injection and embryo transfer}

The mouse ESCs and iPSCs were introduced into early embryos by Piezo micromanipulation as previously described (Huang et al., 2008; Kawase et al., 2001). For the generation of F0 iPSC-derived mice, 10 or 20 iPSCs were injected into 4-cell stage embryos. For ESC contribution assays, 15 ESCs were injected into the blastocyst treated by Activin A as described above. The same numbers of ESCs were injected into embryos in the control group.

CD1 females mated with vasectomized CD1 males were used as pseudopregnant mice. Embryos with injected ESCs were transferred into the uterus or oviduct of pseudopregnant mice, depending on the developmental stage. Blastocysts were transferred into the uterus of pseudopregnant females at 2.5 days post coitum (dpc). Embryos at the morula stage were transferred into the oviduct of $0.5 \mathrm{dpc}$ recipients. We transferred 16-20 embryos per recipient.

\section{ACCESSION NUMBERS}

Raw reads of mouse blastocyst transcriptome data have been submitted to the NCBI Sequence Read Archive (SRA; http://www. ncbi.nlm.nih.gov/sra/) under accession number SRA561196.

\section{ACKNOWLEDGMENTS}

This work was supported by The National Key Research and Development Program of China (2016YFA0100202), National Natural Science Foundation of China (Grant Nos. 31571497 and 
31601941), Beijing Natural Science Foundation of China (6152004), The National Thousand Talents Program of China and Research Programs from the State Key Laboratory for Agrobiotechnology, China Agricultural University (grant numbers 2015SKLAB1-4, 2017SKLAB1-2). The authors acknowledge Dr. Andras Nagy, Kristina Vintersten, and Marina Gertsenstein in Mount Sinai Hospital for their support of R1 and G4-DsRed mouse ESCs. We also wish to thank members of our laboratories for their support.

\section{ABBREVIATIONS}

EPI, epiblast; ESCs, embryonic stem cells; ES-mice, nearly $100 \%$ ESC-derived mice; ICM, inner cell mass; iPSCs, induced pluripotent stem cells; iPS-mice, nearly $100 \%$ iPSC-derived mice; PE, primitive endoderm; PSCs, pluripotent stem cells; TE, trophectoderm; TGF $\beta$, transforming growth factor $\beta$.

\section{COMPLIANCE WITH ETHICS GUIDELINES}

Jinzhu Xiang, Suying Cao, Liang Zhong, Hanning Wang, Yangli Pei, Qingqing Wei, Bingqiang Wen, Haiyuan Mu, Shaopeng Zhang, Liang Yue, Genhua Yue, Bing Lim, and Jianyong Han declare that they have no conflict of interest. All institutional and national guidelines for the care and use of laboratory animals were followed. This article does not contain any studies with human subjects performed by the any of the authors.

\section{AUTHOR CONTRIBUTIONS}

JH. conceived the study and designed the experiments. J.X. performed most of the experiments. S.C. conducted embryonic injection assay for F0 mice generation. L.Z. analyzed the RNA-seq and LC-MS/MS data. H.W. performed ESCs culture, analyzed outgrowths and processed data. Q.W. designed the primers; Y.P. prepared the samples for LC-MS/MS. B.W., S.Z., L.Y. and H.M. helped mouse and embryo preparing works. G.Y. performed the microsatellite analysis. B.L. conducted generation of the iPSCs. J.X. and J.H. wrote the paper.

\section{OPEN ACCESS}

This article is distributed under the terms of the Creative Commons Attribution 4.0 International License (http://creativecommons.org/ licenses/by/4.0/), which permits unrestricted use, distribution, and reproduction in any medium, provided you give appropriate credit to the original author(s) and the source, provide a link to the Creative Commons license, and indicate if changes were made.

\section{REFERENCES}

Cockburn K, Rossant J (2010) Making the blastocyst: lessons from the mouse. J Clin Invest 120:995-1003

Eakin GS, Behringer RR (2003) Tetraploid development in the mouse. Dev Dyn 228:751-766
Evans MJ, Kaufman MH (1981) Establishment in culture of pluripotential cells from mouse embryos. Nature 292:154-156

Fan X, Zhang X, Wu X, Guo H, Hu Y, Tang F, Huang Y (2015) Singlecell RNA-seq transcriptome analysis of linear and circular RNAs in mouse preimplantation embryos. Genome Biol 16:148

Ghimire S, Heindryckx B, Van der Jeught M, Neupane J, O'Leary T, Lierman S, De Vos WH, de Sousa Chuva, Lopes S, Deroo T, De Sutter $P$ (2015) Inhibition of transforming growth factor beta signaling promotes epiblast formation in mouse embryos. Stem Cells Dev 24:497-506

Guzman-Ayala M, Ben-Haim N, Beck S, Constam DB (2004) Nodal protein processing and fibroblast growth factor 4 synergize to maintain a trophoblast stem cell microenvironment. Proc Natl Acad Sci USA 101:15656-15660

Han J, Yuan P, Yang H, Zhang J, Soh BS, Li P, Lim SL, Cao S, Tay J, Orlov YL et al (2010) Tbx3 improves the germ-line competency of induced pluripotent stem cells. Nature 463:1096-1100

Heng JCD, Feng B, Han JY, Jiang JM, Kraus P, Ng JH, Orlov YL, Huss M, Yang L, Lufkin T et al (2010) The nuclear receptor Nr5a2 can replace Oct4 in the reprogramming of murine somatic cells to pluripotent cells. Cell Stem Cell 6:167-174

Huang J, Deng K, Wu H, Liu Z, Chen Z, Cao S, Zhou L, Ye X, Keefe DL, Liu $L$ (2008) Efficient production of mice from embryonic stem cells injected into four- or eight-cell embryos by piezo micromanipulation. Stem Cells 26:1883-1890

Humiecka M, Krupa M, Guzewska MM, Maleszewski M, Suwinska A (2016) ESCs injected into the 8-cell stage mouse embryo modify pattern of cleavage and cell lineage specification. Mech Dev 141:40-50

Inman GJ, Nicolas FJ, Callahan JF, Harling JD, Gaster LM, Reith AD, Laping NJ, Hill CS (2002) SB-431542 is a potent and specific inhibitor of transforming growth factor-beta superfamily type I activin receptor-like kinase (ALK) receptors ALK4, ALK5, and ALK7. Mol Pharmacol 62:65-74

Kang L, Wu T, Tao Y, Yuan Y, He J, Zhang Y, Luo T, Kou ZH, Gao SR (2011) Viable mice produced from three-factor induced pluripotent stem (iPS) cells through tetraploid complementation. Cell Res 21:546-549

Kawase Y, Iwata T, Watanabe M, Kamada N, Ueda O, Suzuki H (2001) Application of the piezo-micromanipulator for injection of embryonic stem cells into mouse blastocysts. Contemp Top Lab Anim Sci 40:31-34

Maherali N, Sridharan R, Xie W, Utikal J, Eminli S, Arnold K, Stadtfeld M, Yachechko R, Tchieu J, Jaenisch R et al (2007) Directly reprogrammed fibroblasts show global epigenetic remodeling and widespread tissue contribution. Cell Stem Cell 1:55-70

Martin GR (1981) Isolation of a pluripotent cell line from early mouse embryos cultured in medium conditioned by teratocarcinoma stem cells. Proc Natl Acad Sci USA 78:7634-7638

Ngangan AV, Waring JC, Cooke MT, Mandrycky CJ, McDevitt TC (2014) Soluble factors secreted by differentiating embryonic stem cells stimulate exogenous cell proliferation and migration. Stem Cell Res Ther 5:26

Ohinata Y, Tsukiyama T (2014) Establishment of trophoblast stem cells under defined culture conditions in mice. PLoS ONE 9:e107308

Pauklin S, Vallier L (2015) Activin/Nodal signalling in stem cells. Development 142:607-619 
Poueymirou WT, Auerbach W, Frendewey D, Hickey JF, Escaravage JM, Esau L, Dore AT, Stevens S, Adams NC, Dominguez MG et al (2007) F0 generation mice fully derived from gene-targeted embryonic stem cells allowing immediate phenotypic analyses. Nat Biotechnol 25:91-99

Ramirezsolis R, Davis AC, Bradley A (1993) Gene targeting in embryonic stem-cells. Method Enzymol 225:855-878

Rossant J, Tam PP (2009) Blastocyst lineage formation, early embryonic asymmetries and axis patterning in the mouse. Development 136:701-713

Stewart CL (1993) Production of chimeras between embryonic stemcells and embryos. Method Enzymol 225:823-855

Takahashi K, Yamanaka S (2006) Induction of pluripotent stem cells from mouse embryonic and adult fibroblast cultures by defined factors. Cell 126:663-676

Takahashi K, Tanabe K, Ohnuki M, Narita M, Ichisaka T, Tomoda K, Yamanaka S (2007) Induction of pluripotent stem cells from adult human fibroblasts by defined factors. Cell 131:861-872

Thomas KR, Capecchi MR (1987) Site-directed mutagenesis by gene targeting in mouse embryo-derived stem-cells. Cell 51:503512
Vintersten K, Monetti C, Gertsenstein M, Zhang P, Laszlo L, Biechele S, Nagy A (2004) Mouse in red: red fluorescent protein expression in mouse ES cells, embryos, and adult animals. Genesis 40:241-246

Wang H, Dey SK (2006) Roadmap to embryo implantation: clues from mouse models. Nat Rev Genet 7:185-199

Wernig $M$, Meissner A, Foreman R, Brambrink T, Ku MC, Hochedlinger K, Bernstein BE, Jaenisch R (2007) In vitro reprogramming of fibroblasts into a pluripotent ES-cell-like state. Nature 448:318

Yousef H, Conboy MJ, Mamiya H, Zeiderman M, Schlesinger C, Schaffer DV, Conboy IM (2014) Mechanisms of action of hESCsecreted proteins that enhance human and mouse myogenesis. Aging 6:602-620

Zernicka-Goetz M, Morris SA, Bruce AW (2009) Making a firm decision: multifaceted regulation of cell fate in the early mouse embryo. Nat Rev Genet 10:467-477

Zhao XY, Li W, Lv Z, Liu L, Tong M, Hai T, Hao J, Guo CL, Ma QW, Wang $L$ et al (2009) iPS cells produce viable mice through tetraploid complementation. Nature 461:86 